\title{
Light-Induced Space-Charge Accumulation Zone as Photovoltaic Mechanism in Perovskite Solar Cells
}

\author{
Isaac Zarazua ${ }^{1}$, Juan Bisquert ${ }^{1,2, *}$ and Germà Garcia-Belmonte ${ }^{1, *}$ \\ ${ }^{1}$ Institute of Advanced Materials (INAM), Universitat Jaume I, 12006 Castelló, Spain \\ ${ }^{2}$ Department of Chemistry, Faculty of Science, King Abdulaziz University, Jeddah, \\ Saudi Arabia
}

Email: bisquert@uji.es, garciag@uji.es

\begin{abstract}
We fabricated formamidinium lead iodide perovskite solar cell for analysis of the photovoltaic mechanism based on the interpretation of the capacitance variation under illumination. It was shown that the low frequency capacitance increases proportional to incident light intensity, and in addition it increases proportional to absorber thickness. Furthermore the voltage dependence of capacitance is exponential with slope $1 / 2 \mathrm{kT}$. We conclude that the large photovoltage and capacitance are associated to electronic accumulation zone at the interface with the metal oxide contact. While this type of accumulation capacitance is common in many devices as transistors, the perovskite solar cell shows a singular behavior in that under light, the electronic carrier accumulation grows unlimited by another series capacitance, reaching values as large as $10 \mathrm{mF} \mathrm{cm}^{-2}$ at one sun illumination.
\end{abstract}

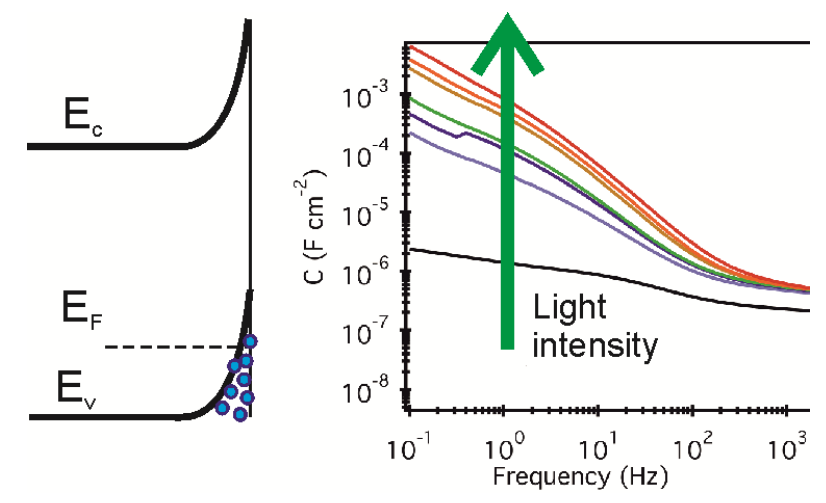

Published in J. Phys. Chem. Lett. 2016, 7, 525-528 
Lead halide organic-inorganic perovskite solar cells have demonstrated a rapid raise of solar energy conversion efficiency that so far exceeded $20 \%{ }^{1-4}$ and is expected to further increase. The hybrid perovskites also show exceptional optoelectronic properties that promise the development of new applications. ${ }^{5,6}$ However, the emergence and widespread observations of peculiar phenomena, including acute hysteresis in current density-voltage $(J-V)$ curve, and switchability by voltage pretreatment, ${ }^{7}$ indicate that the mechanisms that determine the performance of hybrid perovskite solar cells have not yet been understood. Here, we report the interpretation of the large capacitance under illumination ${ }^{8,9}$ that reveals the origin of this feature. We suggest a large accumulation of majority carrier at the contact that indicates an unprecedented photovoltaic mechanism in the family of high performance solar cells.

Previous studies of impedance spectroscopy pointed out the significance of contacts to understand capacitive features of the solar cell device. ${ }^{10,11}$ However, it has now been recognized that hybrid perovskite solar cells contain different kinds of charge carriers: ionic and electronic. Ionic defects can easily drift under internal electrical field and they accumulate to the interfaces. ${ }^{12}$ A large low-frequency capacitance observed in dark conditions has been attributed to ionic electrode polarization, ${ }^{13}$ and this effect has emerged as a dominant factor for time dependent and hysteresis behavior of the lead halide perovskite solar cell. ${ }^{9,14}$ In addition to the capacitive properties in dark, the low frequency capacitance increases rapidly under photogeneration of carriers. ${ }^{8}$ It is therefore reasonable to assume that such capacitance is enhanced by electronic phenomena, requiring a different explanation from the ionic polarization observed in dark conditions. Overall, the large low-frequency capacitance observed to rise under illumination is believed to play a major role in performance via contact modification, ${ }^{15}$ but a detailed understanding of the mechanism, whereby the capacitance displays such extraordinary rise in a solar cell, has not been stated before.

The accumulation of ions at the interface has been reported in a previous paper as origin of capacitive response. ${ }^{13}$ However, a large rise of ionic space charge capacitance is limited by the Helmholtz capacitance situated in series, so that one does not expect values substantially higher than $\mu \mathrm{F} \mathrm{cm}{ }^{-2}$. We fabricated $\mathrm{CH}\left(\mathrm{NH}_{2}\right)_{2} \mathrm{PbI}_{3-x} \mathrm{Cl}_{x}\left(\mathrm{FAPbI}_{3-}\right.$ ${ }_{x} \mathrm{Cl}_{x}$ ) perovskite solar cells of planar structure by the method described in the Experimental part. The values of capacitance vs. frequency shown in Fig. 1 have been measured in open-circuit conditions at different illumination intensities and room temperature. This experimental procedure maximizes charge accumulation as dc currents are suppressed. The high-frequency part is dominated by dielectric and series resistance contributions (see Fig. S1). The low-frequency capacitance increase greatly exceeds the mentioned Helmholtz capacitance limit, as already established before. ${ }^{8}$ Therefore we need to turn to another explanation, in which electronic carriers form a large interfacial capacitance. It is well known that huge electronic interfacial capacitance is possible in the domains of accumulation and inversion at semiconductor surfaces, as there is no limit to the packing of electrons in a short distance at the vicinity of the 
contact. Accumulation capacitance is in fact very common in transistor and MIS devices, ${ }^{16}$ although the measured capacitance is limited by the series connection of the oxide dielectric capacitance. In contrast to this, we suggest that an accumulation capacitance is observed in the perovskite solar cell which is unlimited by any complementary constant capacitance. Accumulation capacitance is however quite uncommon in solar cell devices. This new phenomenon explains the giant capacitance that has been reported previously. ${ }^{8}$

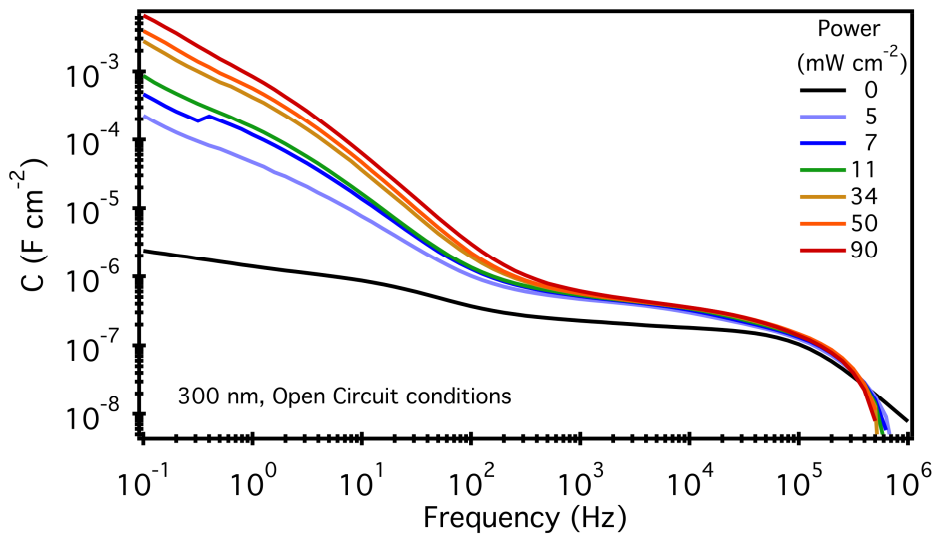

Figure 1. Capacitance spectra measured in open-circuit conditions for different illumination intensities of $300 \mathrm{~nm}$-thick $\mathrm{CH}\left(\mathrm{NH}_{2}\right)_{2} \mathrm{PbI}_{3-x} \mathrm{Cl}_{x}$-based solar cells between 100 $\mathrm{mHz}$ and $1 \mathrm{MHz}$. Dark response at zero-bias is also shown.

To summarize the physical basis of accumulation capacitance, in the $p$-type semiconductor surface, the conduction band bends upward at the surface and penetrates the Fermi level, as shown in Fig. 2c. The charge per unit surface is $Q=q p_{b} L_{D} F_{s}{ }^{17}$ where $q$ is electron charge, $p_{b}$ is the bulk carrier density corresponding to dopant density, $F_{s} \approx \sqrt{2} e^{q V / 2 k_{B} T}$, where $k_{B} T$ is thermal energy and $q V$ corresponds to local separation of vacuum level (VL) from bulk value. $V_{o c}$ is the voltage at the contact as indicated in Fig. 2. The Debye length is given by $L_{D}{ }^{2}=\varepsilon_{r} \varepsilon_{0} k_{B} T / q^{2} p_{b}$ in terms of relative dielectric constant $\varepsilon_{r}$ and vacuum permittivity $\varepsilon_{0}$. For the capacitance we find the expression

$$
C_{s}=\frac{d Q}{d V}=\frac{q p_{b}{ }^{1 / 2}}{\sqrt{2}}\left(\frac{\varepsilon_{r} \varepsilon_{0}}{k_{B} T}\right)^{1 / 2} e^{q V / 2 k_{B} T}
$$


(a)

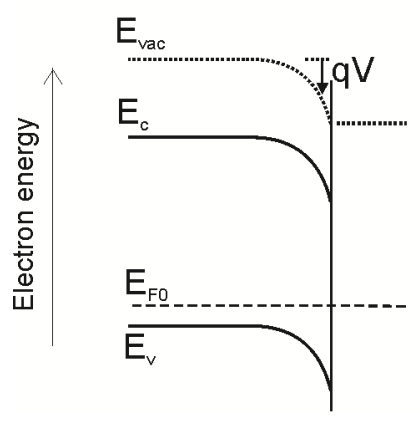

(b)

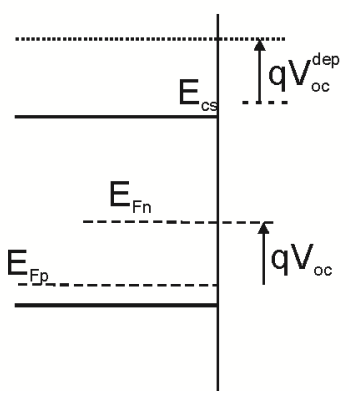

(c)

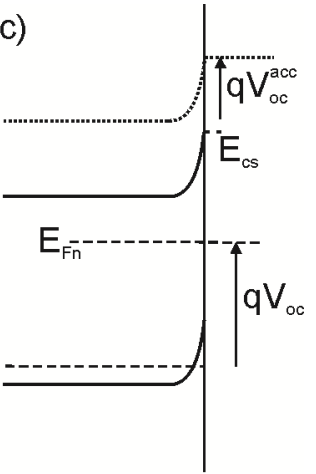

Figure 2. Energy diagram for electron selective contact to the semiconductor absorber layer at open circuit conditions. (a) Depletion layer at equilibrium. Generation of minority carriers produces flatband condition (b) and further accumulation (c) of majority carrier. The total open circuit voltage is the increase of minority carrier Fermi level that corresponds to total change of surface vacuum level as $V_{o c}=V_{o c}^{d e p}+V_{o c}^{a c c}$.

In the planar perovskite solar cell, we assume, as shown in Fig. 2, that voltage drop corresponding to the photovoltage is established at the $\mathrm{TiO}_{2} /$ perovskite interface (equivalent to the potential at the cathode metal), while the spiro-OMeTAD/perovskite hole extraction contact is considered ohmic. This is in agreement with the main charge accumulation interface as observed in transient decays. ${ }^{18}$ In dark equilibrium a depletion layer is observed at this interface as revealed by Mott-Schottky plot and direct measurement of VL. ${ }^{19}$ The increase of minority carriers first removes the depletion region, up to flatband condition, Fig. $2 b$, and then is followed by the creation of accumulation region, Fig. 2c. The accumulation capacitance can grow to very large values, see Fig. S3.

Upon illumination in open circuit photogenerated holes are collected at the accumulation zone in order to build up the measured photovoltage. The absorption depth of formamidinium perovskite is a few hundred nanometers, ${ }^{20}$ greater than the film thickness range (130-300 nm) used here. Consequently, thicker films generate larger amounts of carriers, which are eventually collected at the accumulation zone. The electronic accumulation model therefore predicts an increase of the surface capacitance as a function of the perovskite absorber layer thickness. This is in accordance to the higher open-circuit voltage exhibited by thicker perovskite layers as observed in the $J-V$ curves (Fig. S4). To check such a hole-accumulation mechanism, capacitance per unit length is plotted as a function of the light intensity in Fig. 3a for different layer thickness. Low-frequency capacitance values increases proportionally with the light intensity, as reported previously, ${ }^{8}$ and are also proportional to the perovskite layer thickness. The observation of these two proportionalities (light intensity and film thickness) constitutes a direct proof that the surface capacitance charging is made up of photogenerated carriers coming from the absorber bulk. 

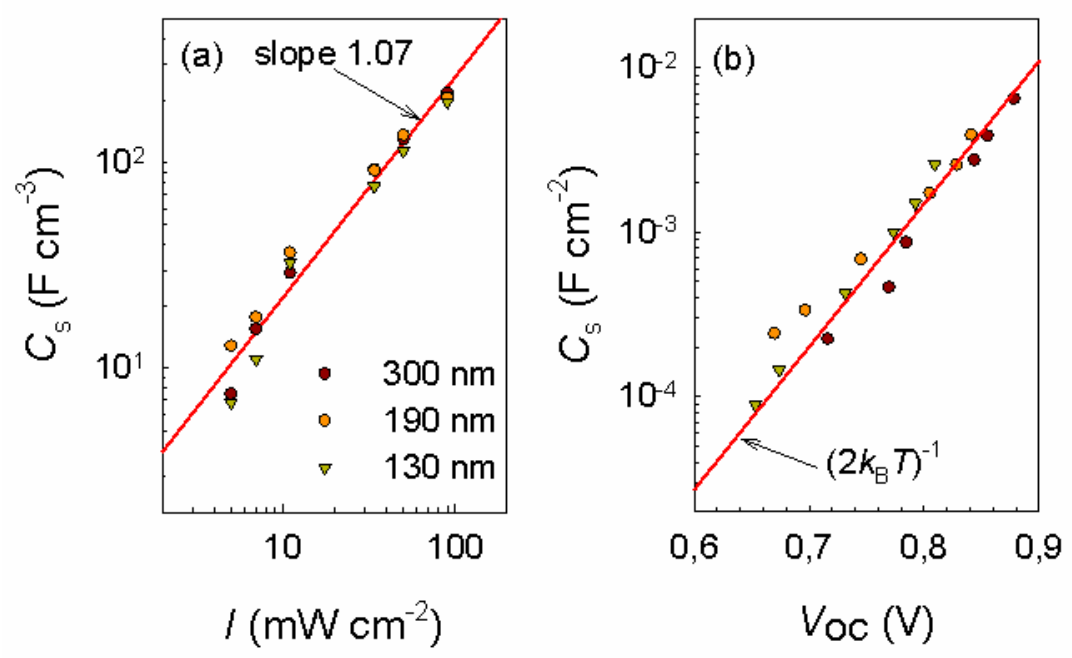

Figure 3. Accumulation capacitance of $\mathrm{FAPbI}_{3-x} \mathrm{Cl}_{x}$-based solar cells extracted from the low-frequency values in Fig. 1. (a) Normalized to the absorber film thickness as a function of the light intensity showing a proportional dependence with the perovskite layer thickness and light intensity, and (b) per unit surface as a function of the opencircuit voltage. Solid line accounts for the fitting using Eq. (1) with exponential dependence on voltage with slope $1 / 2 k_{B} T$ (room temperature) as indicated. Fitting parameters are $p_{b}=2 \times 10^{17} \mathrm{~cm}^{-3}$, and $\varepsilon_{r}=26$.

Experimental capacitance data in Fig. 3b show the characteristic slope of $1 / 2 k_{\mathrm{B}} T$ for solar cells with different absorber perovskite thickness, in agreement with the accumulation capacitance expression (1). The fitting assumes $p_{b}=N_{\mathrm{A}}=2 \times 10^{17} \mathrm{~cm}^{-3}$ as derived from previous works, ${ }^{19}$ and $\varepsilon_{r}=26$ extracted from fitting the capacitance spectra (see Fig. S2). The resulting Debye length is $L_{D}=14 \mathrm{~nm}$, a value non-negligible in relation to the absorber thickness. From a fit (Fig. 2b) the voltage needed to reach flat-band potential under illumination can be estimated which amounts $0.44 \mathrm{~V}$. This value situates $E_{F p}$ at approximately $0.25 \mathrm{eV}$ from the perovskite valence band, consistent with a doping level around $10^{17} \mathrm{~cm}^{-3}$. We note that this voltage does not correspond to the built-in usually encountered through Mott-Schottky analysis performed in the dark, $V_{b i} \approx 1 \mathrm{~V} .{ }^{19}$

Finally an important additional feature should be discussed. Depletion capacitance or accumulation capacitance are different types of dielectric capacitances that respond in short time and high frequency. The typical large low frequency observed in perovskite solar cells is onset at "slow" timescales with frequencies below $100 \mathrm{~Hz}$, see Fig. 1. This type of low frequency accumulation capacitance is quite unexpected and indicates that polarization is dominated by an additional factor that regulates the formation of electronic space charge. Clearly, the large accumulation capacitance is facilitated by large doping density $p_{b}$ as indicated in Eq. (1). We suggest that local doping at the 
interface is controlled by ionic defects, so that the capacitance needs charge compensation ion formation for the accumulation capacitance to take place. This is a self-doping mechanism that obviously depends on the details of contact materials and surface morphology. Such dependence is in agreement with the rather varied phenomenology of contact capacitance and hysteresis. ${ }^{15,21,22}$ We previously connected the low-frequency capacitance excess measured in dark conditions to the electrode polarization caused by ionic defect piled-up. ${ }^{13}$ This mechanism predicts similar capacitive values independently of the absorber layer thickness as seen in experiments (see Fig. S1). The interplay between ionic and electronic effects suggests the occurrence of an electronic interfacial accumulation with a kinetics controlled by ionic defects. A discussion of the difference types of contacts and bulk perovskite materials lies beyond the scope of this report and will be presented in future work.

In conclusion we have described a charge accumulation mechanism that explains a peculiar type of voltage built-in via electronic carrier accumulation at the interface. This observation is suggested as a distinctive photovoltaic operating principle of hybrid organic-inorganic perovskite solar cells.

\section{Experimental Section}

In this study, $\mathrm{CH}\left(\mathrm{NH}_{2}\right)_{2} \mathrm{PbI}_{3-x} \mathrm{Cl}_{x}$ perovskite is used in a planar structure of the type: $\mathrm{FTO} / \mathrm{TiO}_{2} / \mathrm{FAPbI}_{3-x} \mathrm{Cl}_{x} /$ spiro-OMeTAD/Au. All the studied cells were prepared over FTO glasses $(25 \times 25 \mathrm{~mm}$, Pilkington TEC15, $15 \Omega / \mathrm{sq}$ resistance $)$, which were partially etched with zinc powder and $\mathrm{HCl}(2 \mathrm{M})$ in order to avoid short circuits, obtaining $0.25 \mathrm{~cm}^{2}$ of active electrode area. The substrates were cleaned with soap (Hellmanex) and rinsed with Milli-Q water and ethanol. Then, the sheets were sonicated for $15 \mathrm{~min}$ in a solution of acetone:isopropanol (1:1 v/v), rinsed with ethanol, and dried with compressed air. The substrates were treated in a $\mathrm{UV}-\mathrm{O}_{3}$ chamber for $20 \mathrm{~min}$. The $\mathrm{TiO}_{2}$ layer was deposited onto the substrates by spray pyrolysis at $480{ }^{\circ} \mathrm{C}$, using a titanium diisopropoxidebis(acetylacetonate) (75\% in isopropanol, Sigma-Aldrich) solution diluted in ethanol $(1: 39, \mathrm{v} / \mathrm{v})$, with oxygen as carrier gas. The spray was performed in 4 steps of $12 \mathrm{~s}$, spraying each time $10 \mathrm{~mL}$ (approx.), and waiting $1 \mathrm{~min}$ between steps. After the spraying process, the films were kept at $480{ }^{\circ} \mathrm{C}$ for $5 \mathrm{~min}$. Subsequently, a UV-O $\mathrm{O}_{3}$ treatment was performed for $20 \mathrm{~min}$. The thickness determined by scanning electron microscopy was of approximately of $200 \mathrm{~nm}$ observed in Figure S1 (Supporting Information).

The perovskite precursor solution was prepared by reacting $2.64 \mathrm{mmol}$ of $\mathrm{CH}\left(\mathrm{NH}_{2}\right)_{2} \mathrm{I}$ and $0.88 \mathrm{mmol}$ of $\mathrm{PbCl}_{2}(3: 1$ molar ratio) in $1 \mathrm{~mL}$ of DMF. $100 \mu \mathrm{L}$ of this solution was spin-coated inside a glovebox, spin-coating speeds were 1500, 2500, and $3500 \mathrm{rpm}$, producing perovskite films of 130, 190 and $300 \mathrm{~nm}$ (see Figure S1) After the deposition, the substrate was kept at $100{ }^{\circ} \mathrm{C}$ for $10 \mathrm{~min}$. Next, the substrates were heated at $100{ }^{\circ} \mathrm{C}$ during $90 \mathrm{~min}$ in an oven under air stream. Then, the perovskite films were 
covered with the hole-transporting material (HTM, 300 nm thick) by spin coating at $4000 \mathrm{rpm}$ for $30 \mathrm{~s}$ under air conditions, using $100 \mu \mathrm{L}$ of spiro-OMeTAD solution. The spiro-OMeTAD solution was prepared by dissolving in $1 \mathrm{~mL}$ of chlorobenzene $72.3 \mathrm{mg}$ of (2,2',7,7'-tetrakis(N,N'-di-p-methoxyphenylamine)-9,9'-spiro- bifluorene), $28.8 \mu \mathrm{L}$ of 4-tert-butylpyridine, and $17.5 \mu \mathrm{L}$ of a stock solution of $520 \mathrm{mg} / \mathrm{mL}$ of lithium bis(trifluoromethylsulfonyl)imide in acetonitrile. Finally, $60 \mathrm{~nm}$ of gold was thermally evaporated on top of the device to form the electrode contacts using a commercial Univex 250 chamber, from Oerlikon Leybold Vacuum. Before beginning the evaporation, the chamber was evacuated until pressure of $2 \times 10^{-6}$ mbar. The active electrode area of $0.25 \mathrm{~cm}^{2}$ per pixel is defined by the FTO and the Au contacts. Current density-voltage $J-V$ curves were recorded under AM $1.5100 \mathrm{~mW} / \mathrm{cm}^{-2}$ simulated sunlight (ABET Technologies Sun 2000) previously calibrated with an NREL-calibrated Si solar cell. For the measurement of capacitance spectra as function of the light power intensity, a Gambry 3000 potentiostat was used. The AC voltage perturbation was of 10 $\mathrm{mV}$ at open circuit conditions. Each frequency spectrum was measured ranging between $0.1 \mathrm{~Hz}$ and $1 \mathrm{MHz}$ at a given constant incident power, which was changed between 0 and $90 \mathrm{~mW} / \mathrm{cm}^{2}$.

\section{Supporting Information:}

Calculation of permittivity, calculation of accumulation capacitance, current-voltage curves analysis, SEM analysis, photovoltaic parameters.

\section{Acknowlegments}

The work was supported by Generalitat Valenciana project PROMETEO/2014/020 and MINECO of Spain under project MAT2013-47192-C3-1-R. I. Z. thanks CONACYT for the international fellowship. 


\section{References}

(1) Burschka, J.; Pellet, N.; Moon, S.-J.; Humphry-Baker, R.; Gao, P.; Nazeeruddin, M. K.; Grätzel, M. Sequential deposition as a route to high-performance perovskite-sensitized solar cells, Nature 2013, 499, 316-320.

(2) Liu, M.; Johnston, M. B.; Snaith, H. J. Efficient planar heterojunction perovskite solar cells by vapour deposition, Nature 2013, 501, 395-398.

(3) Kojima, A.; Teshima, K.; Shirai, Y.; Miyasaka, T. Organometal halide perovskites as visible-light sensitizers for photovoltaic cells, Journal of the American Chemical Society 2009, 131, 6050-6051.

(4) Yang, W. S.; Noh, J. H.; Jeon, N. J.; Kim, Y. C.; Ryu, S.; Seo, J.; Seok, S. I. High-performance photovoltaic perovskite layers fabricated through intramolecular exchange, Science 2015, 348, 1234-1237.

(5) Xing, G.; Mathews, N.; Lim, S. S.; Yantara, N.; Liu, X.; Sabba, D.; Grätzel, M.; Mhaisalkar, S.; Sum, T. C. Low-temperature solution-processed wavelength-tunable perovskites for lasing, Nat Mater 2014, 13, 476-480.

(6) Suarez, I.; Juarez-Perez, E. J.; Bisquert, J.; Mora-Sero, I.; MartinezPastor, J. P. Polymer/perovskite amplifying waveguides for active hybrid silicon photonics, Advanced Materials 2015, 27, 6157-6162.

(7) Xiao, Z.; Yuan, Y.; Shao, Y.; Wang, Q.; Dong, Q.; Bi, C.; Sharma, P.; Gruverman, A.; Huang, J. Giant switchable photovoltaic effect in organometal trihalide perovskite devices, Nat Mater 2015, 14, 193-198.

(8) Juarez-Perez, E. J.; Sanchez, R. S.; Badia, L.; Garcia-Belmonte, G.; Gonzalez-Pedro, V.; Kang, Y. S.; Mora-Sero, I.; Bisquert, J. Photoinduced giant dielectric constant in lead halide perovskite solar cells, The Journal of Physical Chemistry Letters 2014, 5, 2390-2394.

(9) Kim, H.-S.; Jang, I.-H.; Ahn, N.; Choi, M.; Guerrero, A.; Bisquert, J.; Park, N.-G. Control of I-V hysteresis in $\mathrm{CH}_{3} \mathrm{NH}_{3} \mathrm{PbI}_{3}$ perovskite solar cell, The Journal of Physical Chemistry Letters 2015, 6, 4633-4639.

(10) Pockett, A.; Eperon, G. E.; Peltola, T.; Snaith, H. J.; Walker, A. B.; Peter, L. M.; Cameron, P. J. Characterization of planar lead halide perovskite solar cells by impedance spectroscopy, open circuit photovoltage decay and intensity-modulated photovoltage/photocurrent spectroscopy, The Journal of Physical Chemistry C 2015, $119,3456-3465$.

(11) Pascoe, A. R.; Duffy, N. W.; Scully, A. D.; Huang, F.; Cheng, Y.-B. Insights into planar $\mathrm{CH}_{3} \mathrm{NH}_{3} \mathrm{PbI}_{3}$ perovskite solar cells using impedance spectroscopy, The Journal of Physical Chemistry C 2015, 119, 4444-4453.

(12) Azpiroz, J. M.; Mosconi, E.; Bisquert, J.; De Angelis, F. Defect migration 
in methylammonium lead iodide and its role in perovskite solar cell operation, Energy \& Environmental Science 2015, 8, 2118-2127.

(13) Almora, O.; Zarazua, I.; Mas-Marza, E.; Mora-Sero, I.; Bisquert, J.; Garcia-Belmonte, G. Capacitive dark currents, hysteresis, and electrode polarization in lead halide perovskite solar cells, The Journal of Physical Chemistry Letters 2015, 6, 1645-1652.

(14) Chen, B.; Yang, M.; Zheng, X.; Wu, C.; Li, W.; Yan, Y.; Bisquert, J.; Garcia-Belmonte, G.; Zhu, K.; Priya, S. Impact of capacitive effect and ion migration on the hysteretic behavior of perovskite solar cells, The Journal of Physical Chemistry Letters 2015, 6, 4693-4700.

(15) Tao, C.; Neutzner, S.; Colella, L.; Marras, S.; Srimath Kandada, A. R.; Gandini, M.; Bastiani, M. D.; Pace, G.; Manna, L.; Caironi, M., et al. 17.6\% stabilized efficiency in low-temperature processed planar perovskite solar cells, Energy \& Environmental Science 2015, 8, 2365-2370.

(16) Sze, S. M. Physics of Semiconductor Devizes, 2nd ed.; John Wiley and Sons: New York, 1981.

(17) Mönch, W. Semiconductor Surfaces and Interfaces; Springer: Berlin, 1993.

(18) O’Regan, B. C.; Barnes, P. R. F.; Li, X.; Law, C.; Palomares, E.; MarinBeloqui, J. M. Optoelectronic studies of methylammonium lead iodide perovskite solar cells with mesoporous $\mathrm{TiO}_{2}$ : separation of electronic and chemical charge storage, understanding two recombination lifetimes, and the evolution of band offsets during $\mathrm{J}-\mathrm{V}$ Hysteresis, Journal of the American Chemical Society 2015, 137, 5087-5099.

(19) Guerrero, A.; Juarez-Perez, E. J.; Bisquert, J.; Mora-Sero, I.; GarciaBelmonte, G. Electrical field profile and doping in planar lead halide perovskite solar cells, Applied Physics Letters 2014, 105, 133902.

(20) Eperon, G. E.; Stranks, S. D.; Menelaou, C.; Johnston, M. B.; Herz, L. M.; Snaith, H. J. Formamidinium lead trihalide: a broadly tunable perovskite for efficient planar heterojunction solar cells, Energy \& Environmental Science 2014, 7, 982.

(21) Correa Baena, J. P.; Steier, L.; Tress, W.; Saliba, M.; Neutzner, S.; Matsui, T.; Giordano, F.; Jacobsson, T. J.; Srimath Kandada, A. R.; Zakeeruddin, S. M., et al. Highly efficient planar perovskite solar cells through band alignment engineering, Energy \& Environmental Science 2015, 8, 2928-2934.

(22) Wojciechowski, K.; Stranks, S. D.; Abate, A.; Sadoughi, G.; Sadhanala, A.; Kopidakis, N.; Rumbles, G.; Li, C.-Z.; Friend, R. H.; Jen, A. K. Y.; Snaith, H. J. Heterojunction modification for highly efficient organic-inorganic perovskite solar 
cells, ACS Nano 2014, 8, 12701-12709. 